\title{
Coincidentally identified case of minor salivary gland adenoid cystic carcinoma
}

\begin{abstract}
Minor salivary gland tumors of the oral cavity are rare. They represent approximately $29 \%$ of all minör salivary gland tumors and the most common intraoral site is hard palate. It is a slow growing but aggressive neoplasm with a tendency for recurrence. In this study we will present a 50 years old female patient who was presented with a mass localized at the right portion of the hard palate and was diagnosed incidentally with Adenoid Cystic Carcinoma. In the follow up, no recurrence was noted 10 years after the surgical treatment. Adenoid cystic carcinoma is a salivary gland malignancy with unexpected growth and late recurrence. Our aim was to review the treatment choices of the Adenoid Cystic Carcinoma under the light of current data and to express the importance of surgeon's clinical opinion upon the treatment to be applied.
\end{abstract}

Keywords: adenoid cystic carcinoma, minor salivary gland, hard palate, treatment
Volume 7 Issue 6 - 2017

\author{
Aylin G I,' Mehmet Birol Uur, ${ }^{2}$ Rifat Karli, ${ }^{3}$ \\ Fikret Cnar,' Lokman Uzun ${ }^{4}$ \\ 'Department of Otorhinolaryngology, Bilim University School of \\ Medicine, Turkey \\ ${ }^{2}$ Department of Otorhinolaryngology, Gazi University School of \\ Medicine, Turkey \\ ${ }^{3}$ Department of Otorhinolaryngology, Ondokuz May?s \\ University School of Medicine, Turkey \\ ${ }^{4}$ Department of Otorhinolaryngology, Istanbul Medeniyet \\ University, Turkey
}

\author{
Correspondence: Aylin Gul, Department of \\ Otorhinolaryngology, Dicle University, School of Medicine, \\ Diyarbakir 21280, Turkey, Tel 9041224880014345 , Fax 90412 \\ 2488523,Email draylingul@gmail.com
}

Received: June 01, 2017 | Published: August 03, 2017

\section{Introduction}

Primary tumors of the minor salivary glands are rare and can be seen in palate, buccal mucosa, tongue, lip, paranasal sinuses, pharynx and larynx. Adenoid Cystic Carcinoma (ACC) (or Cylindroma) is the most common cancer of the minor salivary glands. ${ }^{1}$ The typical localisation of the tumor is palate, particularly the junction of the soft and hard palate. ${ }^{2}$ It consist of $1-2 \%$ of all head and neck malignant neoplasms and $10-15 \%$ of all salivary gland tumors. ${ }^{3}$ It is commonly seen in women at 5th- 6th decades. ${ }^{4}$ Typical features of ACC's are indolance, slow growth, local recurrence, perineural invasion and potential of distant metastases. ${ }^{5,6}$ Due to these features, diagnosis and treatment of ACC can be difficult. Surgery, radiotherapy, chemotherapy or combined therapy can be used for ACC treatment.? Surgical resection with wide margins and post operative radiotherapy is the most preferred method for treatment. ${ }^{8}$ Different ratios are reported for tumor recurrence and can be up to $42 \% .{ }^{8}$ In this study, we present a 50 years old female patient who was diagnosed with ACC in her hard palate. We discussed the preferred treatment method of the tumor in the lights of current literature.

\section{Case report}

A fifty years old female patient admitted to our clinic with a sore throat which was persisting for a long time. Physical examination revealed an indolant, fixed, hard consistent mass with a smooth surface and $1 \times 0.5 \mathrm{~cm}$ in size on the right side of the hard palate close to the midline. The patient said she was not aware of this swelling. She said that there was no bleeding, trauma, pain in the area where the swelling was present. Other examination findings were normal. The patient didn't have a story of smoking, alcohol consumption or previous operation. Radiologic scan was normal. Total excision of the mass via transoral approach was planned. Palatal flap was removed under general anesthesia. The mass was totally resected with its fibrous capsule and palatal periosteum beneath it (Figure 1). No bone erosion was detected during operation. Palatal flap was sutured by 3.0 vicryl. Postoperative follow up was uneventful. Pathological evaluation of the mass revealed Adenoid Cystic Carcinoma that did not reach beyond the capsule (Figure 2\&3). Further surgical treatment was discussed for the patient. But, due to the intracapsular limitation of the tumor, only follow up was performed. No local or distant recurrence occured during 10 years of follow up.

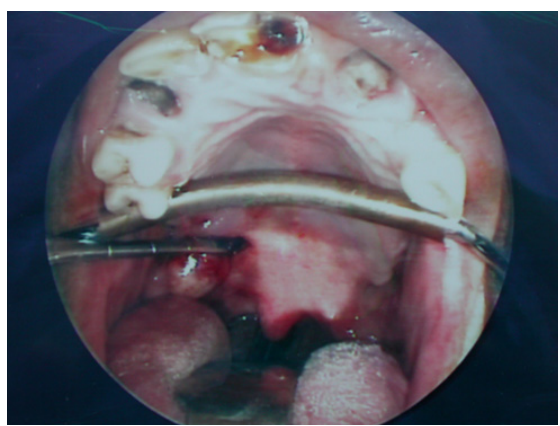

Figure I A mass on the right hard palate close to the midline in a diameter of I $\times 0.5 \mathrm{~cm}$

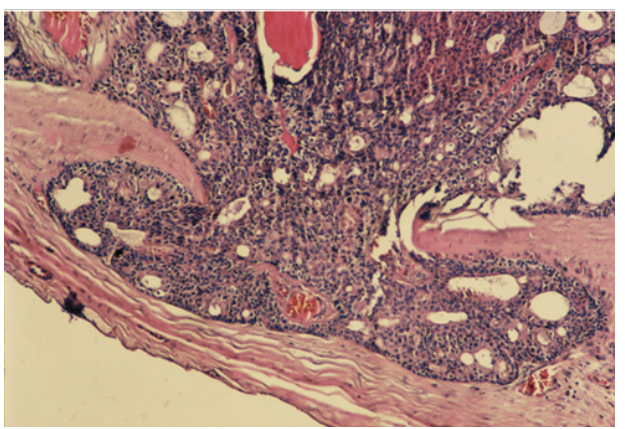

Figure 2 Tumor with a smooth surfaced fibrous capsule. Tumor extends to the capsule but does not exceed the capsule $(H \& E \times 10)$. 


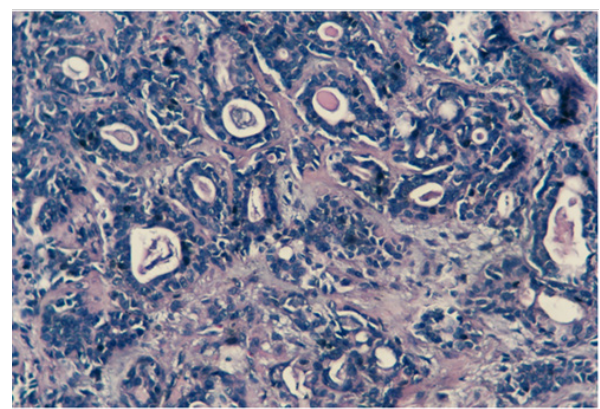

Figure 3 Adenoid cystic structures lined with two rows of atypical cells with eosinophilic sectretions in the lumen (H\&E x20).

\section{Discussion}

ACC is a rare tumor of head and neck region. However, it is the most common malignant tumor of minör salivary glands. ${ }^{1}$ ACC is commonly seen in women at 5th and 6th decades, and demographic features of our patient was similar to the literature data. ${ }^{4} \mathrm{ACC}$ is a slowly growing tumor and has a few years of history prior to the symptoms. In our case, the patient had nonspesific symptoms and the tumor was detected during physical examination. Detection of palatal tumours may be clinically difficult compared to other intraoral sites, which might lead to diagnostic delay and allow extensive neoplasm infiltration consequently resulting in more advanced stages at presentation. ${ }^{9}$ ACCs of the minor salivary glands are submucosal, smooth surfaced, indolent, domed swellings without ulceration. There are 2 clinicsal types of these tumors:

a. Rapidly progressive clinical form develops metastases in short time and leads to death in 2 or 3 years.

b. Natural progressive form has insidious progression and is characterised with a long survey. ${ }^{8}$

Considering the clinical course, we think that our patient was compatible with the latter form. Radiological findings of ACC are non spesific. Perineural invasion and extentions in the soft tissue are best visualised by Magnetic Resonance Imaging (MRI), while bone involvement can be shown best by Computed Tomography (CT). ${ }^{10}$ $\mathrm{CT}$ is valuable for localisation of the tumor, planning the surgical approach and postoperative follow up. ${ }^{11}$ In our patient, preoperative Paranasal CT revealed non spesific findings. ACCs are slow growing tumors. But they have high recurrence rates and may develop distant metastases. So, radical surgical resection with wide margins is performed for treatment of these tumors. ${ }^{7-12}$ There are conflicting reports on the role of postoperative radiotherapy in local control of these tumors. ${ }^{13}$ Payal et al reported that, a 28 years old male patient with a $3 \times 4 \mathrm{~cm}$ mass on the hard palate underwent an excisional biopsy and was diagnosed with ACC. They reported that the patient refused postoperative radiotherapy and no local recurrence was detected in the 1st year follow up. ${ }^{8} \mathrm{Li}$ et al. ${ }^{14}$ reported that 48 of the 108 patients were diagnosed with $\mathrm{ACC}$ who underwent surgical treatment due to minor salivary gland derived tumor on the hard palate. They reported that total surgical resection with wide margin is acceptable for primary treatment, while postoperative radiotherapy was recommended for the patients with poorly differentiated tumors, surgical margin positivity and cervical lymphnode metastases. ${ }^{14}$ Cruz Perez et al. ${ }^{15}$ found that 5 and 10 years survival rates were $56.5 \%$ and $32.5 \%$ in head and neck adenoid cystic carcinoma, respectively. ${ }^{15}$ Jang et al. ${ }^{16}$ Overall survival of 70 patients identified at 5, 10, and 15 years was $80.4 \%, 61.3 \%$, and $29.4 \%$, respectively. Disease recurrence was seen in $31.9 \%$; of these, $72.7 \%$ developed distant metastasis. Older age, higher stage, skull base involvement, positive margins, and metastatic disease, but not local recurrence, predicted a worse overall survival. ${ }^{16}$ Our patient didn't undergo extended surgical resection or radiotherapy because of the small size of the tumor and negative surgical margin. The patient was followed up closely. We think that our treatment protocol was correct when we consider that there was no local or distant recurrence in 10 years of follow up, and the high postoperative life quality of the patient.

\section{Conclusion}

ACCs are rare salivary gland tumors of head and neck region. We think that, surgeon's clinical opinion plays an important role in deciding the treatment protocol in ACCs due to paradoxal biological behavior of this tumor.

\section{Funding}

None.

\section{Conflicts of interest}

The authors declared that there were no financial closure and conflict interests.

\section{Acknowledgments}

None.

\section{References}

1. Spiro, Huvos, Strong EW. Adenoid cystic carcinoma: Factors influencing survival. Am J Surg. 1979;138(4):579-583.

2. Moore, Burkey, Netterville, et al. Surgical management of minor salivary gland neoplasms of the palate. The Ochsner Journal. 2008;8(4):172-180.

3. Kokemueller, Eckardt, Brachvogel, et al. Adenoid cystic carcinoma of the head and neck a 20 years experience. International Journal of Oral and Maxillofacial Surgery. 2004;33(1):25-31.

4. Waldron, El-Mofty, Gnepp. Tumors of the intraoral minör salivary glands: a demographic and histologic study of 426 cases. Oral Surg Oral Med Oral Pathol. 1988;66(3):323-333.

5. Kowalski, Paulino. Perineural invasion in adenoid cystic carcinoma: its causation/promotion by brain derived neurotrophic factor. Hum Pathol. 2002;33(9):933-936.

6. Spiro, Huvos AG, Strong EW. Adenoid cystic carcinoma of salivary origin: A clinicopathologic study of 242 cases. Am J Surg. 1974;128(4):512-520.

7. Chundru, Amudala, Thankappan, et al. Adenoid Cystic Carcinoma Of Palate: A Case Report And Review Of Literature Dent Res J (Isfahan). 2013;10(2):274-278

8. Tripathi, Nahar, Padmavathi, et al. Adenoid Cysic Carcinoma of the Palate: A Case Report with Review of Literature. Journal of Cancer Science \& Therapy. 2010;2(6):160-162.

9. Hanna Hämetoja, Karoliina Hirvonen, Jaana Hagström, et al. Early stage minor salivary gland adenoid cystic carcinoma has favourable prognosis. Virchows Arch. 2017.

10. Peter Hugh. Head and neck imaging. 4th ed, USA: Mosby, Missouri; 2003. p. 1496-1497.

11. Bárbara Vanessa de BM, Rafael Grotta G, Daliana Queiroga de CG, et al. Adenoid Cystic Carcinoma Mimicking an Oroantral Fistula: A Case Report. Int Arch Otorhinolaryngol. 2014;18(2):221-225. 
12. Dequanter, Andry, Lothaire, et al. Wide localized excision and reconstruction for minor salivary gland tumours. B-ENT. 2005;1(4):187-190.

13. Luksic, Suton, Macan, et al. Intraoral adenoid cystic carcinoma: is the presence of perineural invasion associated with the size of the primary tumour, local extension, surgical margins, distant metastases, and outcome? British Journal of Oral and Maxillofacial Surgery. 2014;52(3):214-218.

14. Li Q, Zhang XR, Liu XK, et al. Long-term treatment outcome of minor salivary gland carcinoma of the hard palate. Oral Oncol. 2012;48(5):456-462.
15. Da Cruz Perez, de Abreu Alves, Nobuko Nishimoto, et al. Prognostic factors in head and neck adenoid cystic carcinoma. Oral Oncol. 2006;42(2):139-146.

16. Jang S, Patel PN, Kimple RJ, et al. Clinical Outcomes And Prognostic Factors Of Adenoid Cystic Carcinoma Of The Head And Neck Anticancer Research. Anticancer Res. 2017;37:3045-3052. 